\title{
Efficacy and safety of stereotactic radiosurgery for glomus jugulare tumors
}

\author{
Michael LiM, M.D., Iris C. GibBS, M.D., John R. Adler JR., M.D., \\ and Steven D. Chang, M.D. \\ Departments of Neurosurgery and Radiation Oncology, Stanford University Medical Center, \\ Stanford, California
}

\begin{abstract}
Object. Since the mid-1990s the use of radiosurgery for glomus jugulare tumors has grown in popularity. Despite its increased use, follow-up periods for radiosurgery are short and the numbers of patients reported are small. To add to the available information, the authors report their experience with the application of linear accelerator (LINAC) or CyberKnife modalities in 13 patients with 16 tumors.

Methods. All patients were treated with frame-based LINAC or CyberKnife radiosurgery, with doses ranging from 1400 to $2700 \mathrm{cGy}$. Patients were retrospectively assessed for posttreatment side effects, which included hearing loss, tongue weakness, and vocal hoarseness. The patients' most recent magnetic resonance (MR) images were also assessed for changes in tumor size.

The median follow-up duration was 41 months and the mean follow-up period was 60 months. All tumors remained stable or decreased in size on follow-up MR images. All patients had stable neurological symptoms, and one experienced transient ipsilateral tongue weakness and hearing loss, both of which subsequently resolved. One patient experienced transient ipsilateral vocal cord paresis; however, this individual had received previous external-beam radiation therapy.

Conclusions. The authors' findings continue to support radiosurgery as an effective and safe method of treatment for glomus jugulare tumors that results in low rates of morbidity.
\end{abstract}

\section{KEY WORDS • glomus jugulare tumor • radiosurgery • linear accelerator • CyberKnife}

Paragangliomas make up $0.6 \%$ of all cranial neoplasms. According to Bickerstaff, et al., ${ }^{3}$ these tumors were first described in 1840 by Valentin, who originally characterized the lesion as a ganglion. The term glomus jugulare was coined in 1941 by Guild. ${ }^{11}$ These tumors arise along the glossopharyngeal (Jacobson) nerve or the vagus (Arnold) nerve. Glomus tumors also contain chromaffin cells, which explains their potential to secrete catecholamines. ${ }^{4}$ Clinically, patients can present with deficits of the ninth, 10th, and 11th cranial nerves, or with thrombus of the nearby venous structures or labile blood pressures. ${ }^{4,18}$

As discussed in Lundgren, ${ }^{18}$ the first reported exploration of the jugular bulb for a glomus tumor was performed by Seiffert in 1934 in a patient who presented with a jugular foramen syndrome. Historically, resection has resulted in a high complication rate, which includes stroke (8-20\%), cranial nerve injury (33-44\%), and an overall mortality rate of 5 to $13 \% .{ }^{4}$ After years of experience and with improved microsurgical techniques, complication

Abbreviations used in this paper: GKS = gamma knife surgery; LINAC $=$ linear accelerator; $\mathrm{MR}=$ magnetic resonance. rates are substantially lower, with investigators reporting stroke rates of 0 to $3.5 \%$, cranial nerve injury rates between 5 and $39 \%$, and an overall mortality rate between 0 and 2.7\%. ${ }^{18-21}$ Despite the use of combined approaches, the anatomy of critical cranial nerves and vessels can necessitate a subtotal resection (in as many as $14 \%$ of cases, as reported by Al Mefty and Teixeira ${ }^{1}$ and in $17 \%$ of cases according to Patel, et al. ${ }^{21}$ ). Although not all tumors with subtotal resections continue to grow, this subgroup of patients may require further surgery, embolization, or radiation.

Treatment of glomus jugulare tumors was initially supplemented with external-beam radiation therapy. Although tumor control rates were high, patients reported complications such as skin changes and dry mouth. Radiosurgery to treat these tumors was introduced in the mid1990s. ${ }^{14}$ Despite this advance, the use of radiosurgery for these lesions has been reported in only a few centers, and the series sizes have been small, with only short-term follow-up periods. We report our experience in treating glomus jugulare tumors with stereotactic radiosurgery, examining both the efficacy and side effects of treatment. 


\section{CLINICAL MATERIAL AND METHODS}

Before we began the study, the Stanford Internal Review Board committee first approved the proposed patient treatment and follow-up protocol. A retrospective analysis of the Stanford University Hospital radiosurgery database was then conducted to identify patients with glomus jugulare tumors who were treated with LINAC or CyberKnife modalities between 1991 and 2004 (Fig. 1). Patient records were examined for the following data: age, sex, laterality of tumor, dose, number of isocenters, pre- and posttreatment symptoms, largest dimension of tumor on MR images before and after therapy, and duration of follow-up review in which neuroimages and clinical symptoms were assessed (Table 1). Clinical symptoms were also documented by telephone interviews, and the patients' MR images were obtained to confirm the findings.

\section{Patient Population}

Patients ranged in age from 21 to 80 years, with a mean age of 52 years. Of the 13 patients included in the study (five men and eight women) there were 16 tumors; two patients had multiple lesions. Four patients had previously undergone open surgeries for their tumors; two of them underwent multiple resections. Nine of the 13 patients were treated exclusively with radiosurgery. Tumor sizes ranged from 1.2 to $6.2 \mathrm{~cm}$ at the largest measurable diameter, with a mean size of $3 \mathrm{~cm}$. Of the five patients who presented with recurring tumors (all previously resected, with histological features consistent with glomus tumors), the lesion extended up to the cavernous sinus in one, whereas the remainder had local recurrences. Presenting symptoms involved the ninth (five patients), 10th (four patients), 11th (four patients), and 12th (two patients) cranial nerves.

Five tumors were treated using a frame-based LINAC system, and the remaining 11 were treated with the CyberKnife. Prescribed doses to the periphery of the tumor (typically to the $80 \%$ isodose line) ranged from 1400 to 2700 cGy. Patients who underwent LINAC radiosurgery were treated with one to three isocenters and those treated with the CyberKnife received nonisocentric-based treatments.

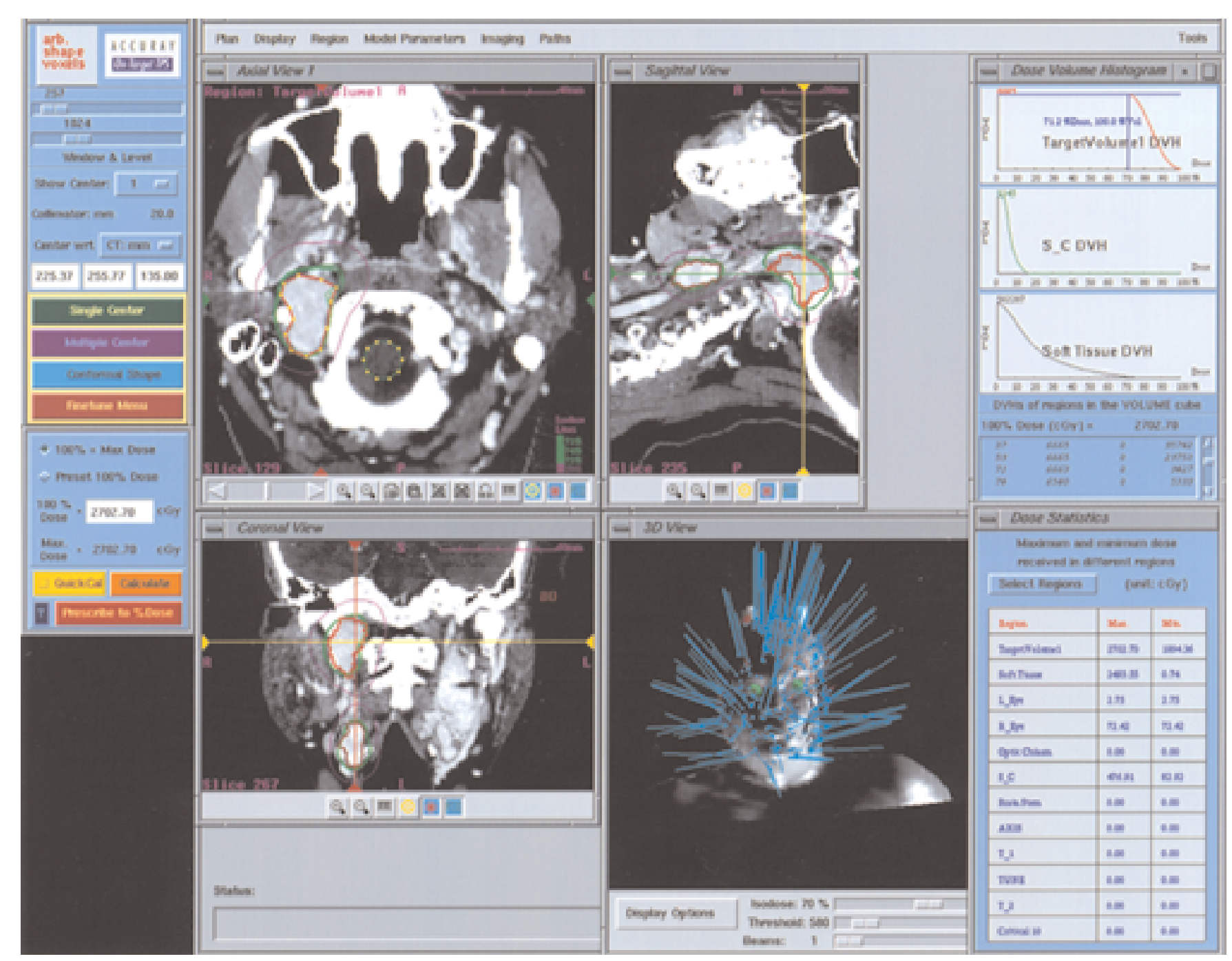

Fig. 1. Photograph of workstation monitor showing example of a treatment plan for a patient with a glomus tumor. 
TABLE 1

Demographic data and duration of neuroimaging and clinical follow-up period in 13 patientswith glomus jugulare tumors*

\begin{tabular}{|c|c|c|c|c|c|c|c|c|}
\hline \multirow{2}{*}{$\begin{array}{l}\text { Case } \\
\text { No. }\end{array}$} & \multirow{2}{*}{$\begin{array}{l}\text { Age (yrs), } \\
\text { Sex }\end{array}$} & \multirow{2}{*}{$\begin{array}{l}\text { Tumor Side } \\
\& \text { Size }(\mathrm{cm})\end{array}$} & \multirow[b]{2}{*}{ Prev Op } & \multirow[b]{2}{*}{ Modality } & \multirow{2}{*}{$\begin{array}{l}\text { Dose } \\
\text { (cGy) }\end{array}$} & \multirow{2}{*}{$\begin{array}{l}\text { No. of } \\
\text { Fractions }\end{array}$} & \multicolumn{2}{|c|}{$\mathrm{FU}(\mathrm{mos})$} \\
\hline & & & & & & & MR Imaging & Clinical \\
\hline 1 & $75, \mathrm{M}$ & 1t, 2.8 & $1.5 \mathrm{yrs}$ & CyberKnife & 2000 & 1 & 55 & 114 \\
\hline $2 \dagger$ & $47, \mathrm{~F}$ & lt, 1.5 & $9 \operatorname{mos}$ & CyberKnife & 1800 & 1 & 24 & 35 \\
\hline 3 & $43, \mathrm{~F}$ & $\mathrm{rt}, 3.6$ & $6,12,18, \& 21 \mathrm{yrs}$ & LINAC & 1600 & 1 & 30 & 73 \\
\hline $4 \dagger$ & $61, \mathrm{M}$ & 1t, 2.5 & none & LINAC & 1800 & 1 & 6 & 6 \\
\hline 5 & $30, \mathrm{~F}$ & $\mathrm{rt}, 2.0$ & none & LINAC & 1800 & 1 & 125 & 172 \\
\hline 6 & $46, \mathrm{~F}$ & $\mathrm{rt}, 1.2$ & $13 \& 14 \mathrm{yrs}$ & LINAC & 2500 & 1 & 139 & 144 \\
\hline 7 & $76, \mathrm{~F}$ & lt, 2.7 & none & LINAC & 2500 & 3 & 126 & 187 \\
\hline $8 \ddagger$ & $45, \mathrm{~F}$ & 1t, 3.8 & none & CyberKnife & 1800 & 1 & 45 & 53 \\
\hline \multirow[t]{2}{*}{9} & $24, \mathrm{M}$ & $\mathrm{rt}, 2.3$ & none & CyberKnife & 1800 & 3 & 46 & 59 \\
\hline & & lt, 1.7 & none & CyberKnife & 1800 & 1 & 43 & 46 \\
\hline \multirow[t]{3}{*}{10} & $52, \mathrm{~F}$ & $1 \mathrm{t}, 6.2$ & none & CyberKnife & 1400 & 1 & 17 & 17 \\
\hline & & $\mathrm{rt}, 2.8$ & none & CyberKnife & 2700 & 1 & 4 & 4 \\
\hline & & $\mathrm{rt}, 3.1$ & none & CyberKnife & 2700 & 1 & 4 & 4 \\
\hline 11 & $85, \mathrm{~F}$ & $\mathrm{rt}, 3.6$ & none & CyberKnife & 1870 & 1 & 12 & 18 \\
\hline $12 \ddagger$ & $37, \mathrm{M}$ & lt, 6.2 & none & CyberKnife & 2400 & 3 & 9 & 9 \\
\hline 13 & $80, \mathrm{~F}$ & lt, 2.2 & none & CyberKnife & 2000 & 1 & 18 & 18 \\
\hline mean & 51.8 & 3.0 & & & & & 44 & 60 \\
\hline median & & & & & & & 27 & 41 \\
\hline
\end{tabular}

$* \mathrm{FU}=$ follow up; prev = previous

$\dagger$ Patients died of a drug overdose (Case 2) and of multiple myeloma (Case 4).

$\ddagger$ Tumor size decreased; unchanged on follow-up review in all cases.

Follow-up durations ranged from 4 to 139 months with neuroimages (mean 44 months, median 27 months) and 4 to 172 months with clinical follow-up visits (mean 60 months, median 41 months).

\section{RESULTS}

After treatment, two patients experienced transient worsening of preoperative cranial nerve deficits: the first noted transient ipsilateral tongue atrophy and hearing loss that both began 2 months posttreatment and resolved over the following 2 months, and the second reported worsened vocal hoarseness (confirmed with laryngoscopy) that began 1 month posttreatment and resolved over 8 months. The remaining patients experienced no side effects.

All 16 tumors were stable at the time of follow-up review of MR images, which ranged from 4 to 139 months (all images were obtained with 5-mm slices and a zero skip gap). Two patients who were treated with stereotactic radiosurgery alone experienced a tumor regression. The patient in Case 8 had a tumor whose largest diameter on neuroimaging studies was $3.8 \mathrm{~cm}$ before treatment, and at 15 months after treatment the lesion had decreased to a maximal diameter of $3.4 \mathrm{~cm}$. The patient in Case 15 had a tumor whose largest diameter on neuroimaging studies was $6.2 \mathrm{~cm}$ before treatment, and at 8 months after treatment the lesion had decreased to a maximal diameter of $5 \mathrm{~cm}$.

\section{DISCUSSION}

Glomus jugulare tumors are indolent, well-vascularized lesions that cause symptoms primarily through mass effect on neighboring structures such as cranial nerves and vasculature. ${ }^{14}$ Historically, surgical treatment for glomus jugulare tumors was thought to result in a high rate of morbidity because of the cranial nerve and vascular structures with which these lesions are intimately associated. In addition, the overall mortality rate was between 5 and $13 \% .{ }^{4}$ With the advent of improved microsurgical techniques and the use of combined approaches, the mortality rate has lowered substantially; however, cranial nerve injury continues to be a problem. ${ }^{18-21}$ In a retrospective series of 176 patients treated between 1972 and 1998 and reported by Jackson, et al., ${ }^{12}$ in $85 \%$ complete surgical control was described. The remaining $15 \%$ of their patients had a subtotal resection or recurrence. Complications included $4.5 \%$ of patients with cerebrospinal fluid leaks; deficits of the ninth $(39 \%)$, 10th $(25 \%), 11$ th $(26 \%)$, and 12 th cranial nerves $(21 \%)$; and a mortality rate of $2.7 \%$. Although the mortality rates have improved significantly, a relatively high rate of cranial nerve dysfunction remains. The higher rate of morbidity, however, may be attributed to the fact that patients treated in 1972 were included in the analysis; the authors indicated that more complications occurred before 1987.

In reporting on a series of 43 patients in 2002 (of whom 28 were surgically treated by the authors), Al-Mefty and Teixeira ${ }^{1}$ claimed that a gross-total resection was achieved in $24(86 \%)$ of 28 patients, with tumor recurrence in only two $(7 \%)$ of 28 . In addition, their patients' mortality rate was $0 \%$. Complications reported in their study included one patient with a hemiplegia from a carotid artery thrombosis that was reversed with urokinase treatment, four patients with cerebrospinal fluid leaks, and five with infection. Nevertheless, new cranial nerve deficits developed in 11 patients $(39 \%)$, with five requiring a tracheostomy and four requiring a gastrostomy at a mean follow-up duration of 38 months. Many of the patients in this series presented with symptomatic, large, complex tumors, and lower rates of cranial nerve deficits may be seen in smaller lesions. 
Green, et al., ${ }^{10}$ however, reported better results for cranial nerve function. In their series of 52 tumors, in which 43 patients were treated surgically via the infratemporal fossa approach, $95 \%$ of their patients had at least a Grade I or II on the House scale with respect to facial nerve function at a mean follow-up time of 4.2 years (note that $88 \%$ of patients presented with a normal [House Grade I] function). As for the other cranial nerves, $23 \%$ of patients presented with a ninth nerve deficit; however, $49 \%$ of their patients had a ninth cranial nerve deficit postoperatively. Although $34 \%$ presented with a 10th cranial nerve deficit, $46 \%$ had a postoperative deficit in the same nerve. Whereas $18 \%$ presented with an 11th cranial nerve deficit, $43 \%$ had a postoperative deficit in this nerve. Lastly, $20 \%$ presented with a 12th cranial nerve deficit and $27 \%$ had a postoperative deficit in this nerve. These authors also reported that $85 \%$ of their patients were able to resume all preoperative activities. Nevertheless, $15 \%$ of their patients had been unable to perform activities of daily living preoperatively.

Patel, et al., ${ }^{21}$ published their results in a series of 12 patients; they achieved complete resection in 10 of them by using combined approaches. These authors again demonstrated no surgically related deaths or episodes of stroke; however, three patients (33\%) required facial nerve resection during the procedure, followed by nerve grafting. Another patient experienced a postoperative hemiparesis from hydrocephalus and brainstem distortion that resolved in the course of 2 years. Only one patient also experienced mild persistent swallowing difficulty. These improvements in surgical techniques indicate that resection is a viable and safe option for many patients and may be the best choice for large tumors with mass effect.

External-beam radiation therapy, which was initially introduced as an adjuvant treatment for subtotal resections, was later used as primary therapy in patients who were not surgical candidates. Investigators in centers in which external-beam radiation therapy was used have reported tumor control (defined as stable or decreased tumor size) in as many as $61 \%$ of their cases, with $71 \%$ of patients reporting improvement of their clinical symptoms. ${ }^{20}$

The drawbacks associated with external-beam radiation therapy are the large field sizes, which include the upper neck and skull base. Reported complications include radionecrosis of bone, possible induction of secondary malignancies, and xerostomia. ${ }^{13,14}$ The introduction of intensity-modulated radiation therapy has reduced the amount of normal tissue exposed to radiation, to avoid the side effects. Despite these drawbacks, the introduction of radiosurgery has offered a new technique to deliver radiation precisely.

Glomus jugulare tumors are ideally suited for radiosurgery; they are well-defined, noninfiltrating lesions, and they usually present with a reasonably small size. Unlike conventional radiation therapy, the steep dose gradient achievable with radiosurgery minimizes irradiation of neighboring normal tissue and allows delivery of a larger dose of radiation to this slowly proliferating tumor without exceeding the radiation tolerance of normal tissues.

Despite the aforementioned benefits of using radiosurgery to treat glomus jugulare tumors, there are issues of precise target delineation. Given the complexity of the neck anatomy, both the LINAC and CyberKnife modalities have small treatment delivery errors $(<2 \mathrm{~mm})$ that translate into potential underdosing of a tumor edge or inclusion of neighboring cranial nerves. Modern neuroimaging cannot reliably separate tumor from adjacent cranial nerves when targeting for radiosurgery treatment, whereas such concerns are less important with external-beam radiation therapy. In addition, although radiosurgery minimizes the dose of radiation that normal neighboring tissue receives, there remains the risk that normal tissue will be exposed to radiation. Permanent cranial nerve deficits are rare, but they are still possible side effects of stereotactic radiosurgery.

In 1995, the first published series on the use of GKS for glomus tumors was reported. ${ }^{14}$ Since then, several additional studies have been reported in the literature..$^{5-9,14,16,17}$ Using GKS, Foote and colleagues ${ }^{8,9}$ treated nine patients with glomus tumors. In all nine, no tumor growth was seen after treatment and no long-term complications were reported. Jordan, et al., ${ }^{14}$ reported on eight patients treated with GKS, noting no tumor progression and no delayed cranial neuropathies. Both groups of investigators remarked on the minimal number of side effects. Liscak, et al., ${ }^{17}$ however, reported a complication from their study of 14 patients treated with radiosurgery. Although this study also showed $100 \%$ control in tumor progression, with all patients reporting at least some clinical improvement, three patients experienced worsening hearing. One must also note that not all groups achieved $100 \%$ control of tumor growth. Recently, Eustacchio, et al., ${ }^{6}$ reported their experience in 19 patients treated using GKS. They reported a $94.7 \%$ tumor control rate, with no complications. In addition, Feigenberg, et al., ${ }^{7}$ only reported tumor control in three of five patients in whom LINAC therapy was administered.

The longest follow-up duration was reported by Bari, et al. ${ }^{2}$ They treated eight patients with glomus jugulare tumors by using GKS. Their follow-up duration was between 52 and 97 months. In their group, all patients were clinically stable and five of the eight had a decrease in the size of the lesion. They cautioned, however, that a 10 -year follow-up period would be more conclusive.

As for the LINAC experience, Feigenberg, et al., ${ }^{7}$ reported on a series of five patients with glomus tumors who were treated with LINAC radiosurgery. Their median follow-up duration was 27 months. Their results were not as encouraging; two of the five tumors had grown at 6 and 40 months after radiosurgery. Nevertheless, their patient numbers were small. In a more recent series by Maarouf, et al., ${ }^{19}$ the authors reported their experience with LINAC therapy in 12 patients with glomus jugulare tumors. In their study the median dose was $15 \mathrm{~Gy}$ and the median follow-up period was 4 years. All patients attained a stable or decreased tumor size with stable or improved clinical symptoms. What was interesting was that at Feigenberg and colleagues' institution a higher median dose of 25 Gy was used, but their tumor control was inferior, even with a shorter follow-up duration. In our earlier experience with the LINAC and CyberKnife modalities, we reported our results in 10 tumors treated with a dose be- 
tween 16 and 25 Gy. Our median follow-up duration was 21.5 months, and we achieved $100 \%$ control of tumor growth and stable clinical symptoms. ${ }^{15}$

As for complications, because of the proximity of glomus jugulare tumors to the lower cranial nerves, we must first consider the susceptibility of these nerves to injury with either surgery or radiosurgery. ${ }^{4}$ An encouraging finding in our study, which has been confirmed in other reports, is the radiation resistance of the cranial nerves. In our series there were two patients with transient cranial nerve deficits that resolved completely. The rate of cranial nerve injury in the majority of the aforementioned studies was also very low. ${ }^{9,14,17}$

As encouraging as these results are, we must still be cautious about the initial studies. As Bari, et al., ${ }^{2}$ have previously emphasized, the indolent growth rate of the glomus jugulare tumor still requires a 10-year follow-up period to determine the treatment's true efficacy. In our three patients who have completed more than 10 years of follow up, they continue to report stable clinical symptoms with no side effects. We are optimistic that outcomes in the more recently treated patients will continue to be good.

\section{CONCLUSIONS}

Our study is the largest single-center series in which a LINAC-based treatment has been used. We report a 100\% control rate of tumors, with stable clinical symptoms. Our results continue to demonstrate that tumor control, preservation of cranial nerves, and minimal side effects are maintained within a larger cohort. Stereotactic radiosurgery remains a safe and efficacious form of treatment for glomus jugulare tumors, and is associated with minimal morbidity.

\section{Acknowledgment}

We thank Beth Hoyte for assistance with production of the figure.

\section{Disclosure}

Dr. Adler is the Chief Medical Officer of and a shareholder in Accuray, Inc., which manufactures the CyberKnife.

\section{References}

1. Al-Mefty O, Teixeira A: Complex tumors of the glomus jugulare: criteria, treatment, and outcome. J Neurosurg 97: 1356-1366, 2002

2. Bari ME, Kemeny AA, Forster DM, et al: Radiosurgery for the control of glomus jugulare tumours. J Pak Med Assoc 53: 147-151, 2003

3. Bickerstaff ER, Howell JS: The neurological importance of tumours of the glomus jugulare. Brain 76:576-593, 1953

4. Chretien PB, Engelman K, Hoye RC, et al: Surgical manage- ment of intravascular glomus jugulare tumor. Am J Surg 122: 740-743, 1971

5. Eustacchio S, Leber K, Trummer M, et al: Gamma knife radiosurgery for glomus jugulare tumours. Acta Neurochir 141: 811-818, 1999

6. Eustacchio S, Trummer M, Unger F, et al: The role of Gamma Knife radiosurgery in the management of glomus jugular tumours. Acta Neurochir Suppl 84:91-97, 2002

7. Feigenberg SJ, Mendenhall WM, Hinerman RW, et al: Radiosurgery for paraganglioma of the temporal bone. Head Neck 24:384-389, 2002

8. Foote RL, Coffey RJ, Gorman DA, et al: Stereotactic radiosurgery for glomus jugulare tumors: a preliminary report. Int J Radiat Oncol Biol Phys 38:491-495, 1997

9. Foote RL, Pollock BE, Gorman DA, et al: Glomus jugulare tumor: tumor control and complications after stereotactic radiosurgery. Head Neck 24:332-339, 2002

10. Green JD Jr, Brackmann DE, Nguyen CD, et al: Surgical management of previously untreated glomus jugulare tumors. Laryngoscope 104:917-921, 1994

11. Guild SR: The glomus jugulare, a nonchromaffin paraganglion, in man. Ann Otol Rhinol Laryngol 62:1045-1071, 1953

12. Jackson CG, McGrew BM, Forest JA, et al: Lateral skull base surgery for glomus tumors: long-term control. Otol Neurotol 22:377-382, 2001

13. Jordan JA, Roland PS, McManus C, et al: Stereotactic radiosurgery for glomus jugulare tumors. Laryngoscope 110:35-38, 2000

14. Kida Y, Kobayashi T, Tanaka T, et al: [A new strategy for the treatment of jugular foramen tumors using radiosurgery.] No Shinkei Geka 23:671-675, 1995 (Jpn)

15. Lim M, Gibbs IC, Adler JR Jr, et al: The efficacy of linear accelerator stereotactic radiosurgery in treating glomus jugulare tumors. Technol Cancer Res Treat 2:261-265, 2003

16. Liscak R, Vladyka V, Simonova G, et al: Leksell gamma knife radiosurgery of the tumor glomus jugulare and tympanicum. Stereotact Funct Neurosurg 70 (Suppl 1):152-160, 1998

17. Liscak R, Vladyka V, Wowra B, et al: Gamma knife radiosurgery of the glomus jugulare tumour-early multicentre experience. Acta Neurochir 141:1141-1146, 1999

18. Lundgren N: Tympanic body tumours in the middle ear. Tumours of carotid body type. Acta Otolaryngol 37:367-379, 1949

19. Maarouf M, Voges J, Landwehr P, et al: Stereotactic linear accelerater-based radiosurgery for the treatment of patients with glomus jugulare tumors. Cancer 97:1093-1098, 2003

20. Mukherji SK, Kasper ME, Tart RP, et al: Irradiated paragangliomas of the head and neck: CT and MR appearance. AJNR 15: 357-363, 1994

21. Patel SJ, Sekhar LN, Cass SP, et al: Combined approaches for resection of extensive glomus jugulare tumors. A review of 12 cases. J Neurosurg 80:1026-1038, 1994

Manuscript received June 15, 2004.

Accepted in final form July 21, 2004.

Address reprint requests to: Steven D. Chang, M.D., Department of Neurosurgery, Stanford University School of Medicine, 300 Pasteur Drive, Room R-225, Stanford, California 94305. email: sdchang@stanford.edu. 